\title{
NMR Diffusometry of Oil-in-Water Emulsions
}

\author{
B.P. Hills*, H-R. Tang, P. Manoj, C. Destruel \\ Institute of Food Research, Norwich Research Park, Colney, Norwich NR4 7UA, UK
}

\begin{abstract}
The diffusion propagator of the continuous aqueous phase of concentrated oil-in-water emulsions is used to probe the relationship between emulsion microstructure and bulk rheological properties. This is done by expanding the stimulated echo amplitude, $\mathrm{S}(\mathrm{q}, \Delta)$, as a multiple exponential time series expansion in $\Delta$, with wavevector dependent expansion coefficients. These coefficients are compared with predictions from several theoretical models for three types of stable emulsion, each differing in microstructure. Empiric relationships between the wavevector expansion coefficients and bulk rheology are established. () 2001 Elsevier Science Inc. All rights reserved.
\end{abstract}

Keywords: NMR; emulsions; water; diffusion; rheology

\section{Introduction}

Processes such as flocculation, droplet coalescence and creaming limit emulsion stability and change rheological properties thereby affecting the shelflife of foods, paints and pharmaceutical preparations. Unfortunately the relationship between emulsion microstructure and bulk rheology remains poorly understood. In this communication we explore this structure-function relationship with NMR q-space diffusometry [1] focusing on the nature of the diffusion propagator of the continuous phase. To analyze this propagator we use a multiple exponential time series expansion of the NMR stimulated echo amplitude as a function of increasing diffusion time, $\Delta$. The coefficients in this expansion are then compared with both analytical models and bulk rheological parameters.

By controlling the coalescence, flocculation and creaming processes at least three types of stable (i.e., time-invariant) model emulsion microstructure can be prepared. The first is a density-matched, non-flocculating, non-coalescing emulsion consisting, essentially, of individual oil droplets undergoing Brownian motion. The second is a non-creaming, non-coalescing density-matched emulsion created by inducing depletion flocculation with the addition of low concentrations of non-adsorbing biopolymer. This type of emulsion contains many flocs or groups of associated oil

\footnotetext{
* Corresponding author.

E-mail address: brian.hills@bbsrc.ac.uk (B.P. Hills).
}

droplets. These flocs are generally believed to form by diffusion limited aggregation and therefore have a fractaltype structure. A third type of spatially uniform, stable emulsion microstructure is found in the upper creamed layer formed when a flocculated or unflocculated emulsion is allowed to cream under gravity. The flocculated cream microstructure will depend on the "flexibility" of the flocs, that is, whether they are sufficiently rigid to retain an open fractal geometry or whether droplets can rearrange within the flocs to form a more thermodynamically stable, closer packed structure.

\section{NMR relaxometry}

It was first established that the transverse and longitudinal relaxation time spectra (i.e., the continuous distribution of relaxation times) derived from the CPMG and inversion recovery sequences were insensitive to emulsion microstructure, at least in the model emulsions used in this study. This is not surprising because the oil and water relaxation time peaks were well resolved and no mechanism, such as proton exchange or spin diffusion, is effective in exchanging magnetization between the two proton pools. Fig. 2 shows a typical relaxation time spectrum. The fact that the oil and water peaks are well separated does, however, permit the oil volume fraction to be derived from the relative peak areas. This rapid, non-invasive measurement proved to be invaluable for determining the oil content of the creamed phase of the flocculated and unflocculated emulsions. The 


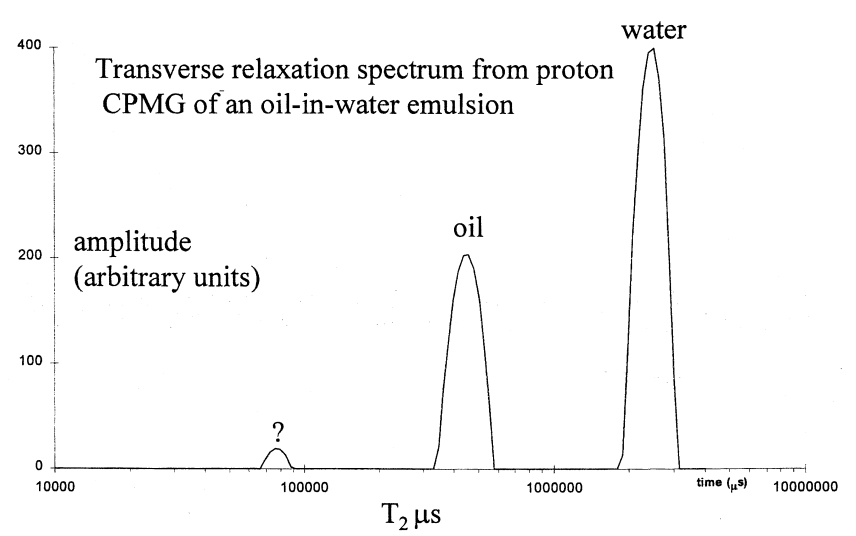

Fig. 1. Transverse relaxation time spectrum for a hexadecane-water emulsion.

small peak at about $80 \mathrm{msec}$ is unassigned, but could arise from surfactant micelles.

\section{NMR diffusometry}

Fig. 3 shows a typical plot of the stimulated echo attenuation plotted in the conventional way against $\mathrm{q}^{2 \Delta}$ for increasing wavevector, q. Unfortunately this way of plotting the data is not the most convenient for our present purposes. To compare the NMR q-space data for the three emulsion types with idealized analytical models and rheological data, it is far easier to plot the stimulated echo amplitude, $\mathrm{S}(\mathrm{q}, \Delta)$ for the continuous aqueous phase as a function of increasing diffusion time, $\Delta$, and expand it as a multiple exponential time series, such that,

$$
\mathrm{S}(\mathrm{q}, \Delta) / \mathrm{S}(0, \Delta)=\sum_{\mathrm{n}=0, \mathrm{~m}} \mathrm{p}_{\mathrm{n}}(\mathrm{q}) \exp \left[-\mathrm{D}_{\mathrm{n}}(\mathrm{q}) \Delta\right]
$$

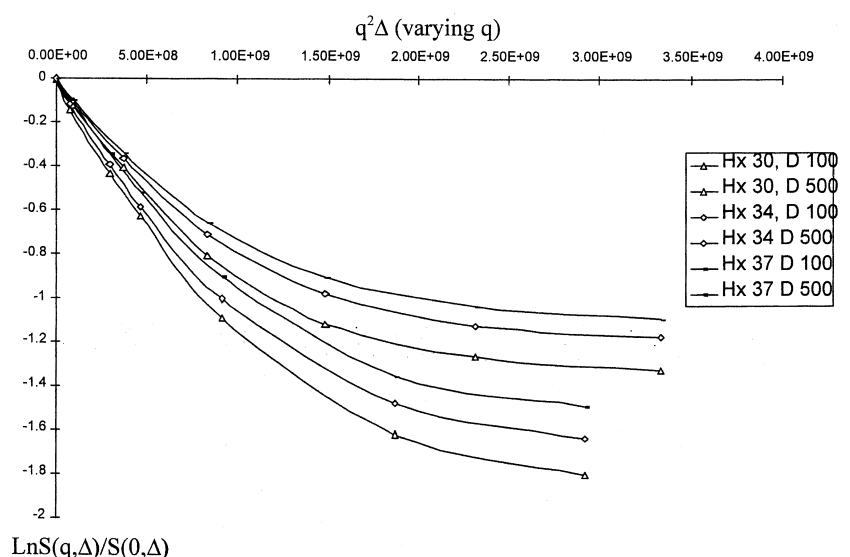

Fig. 2. The stimulated echo attenuation for a flocculated bromohexadecane emulsion for the concentrations and diffusion times (D in milliseconds) indicated.
The amplitudes, $\mathrm{p}_{\mathrm{n}}(\mathrm{q})$ and diffusion rates, $\mathrm{D}_{\mathrm{n}}(\mathrm{q})$ can then be compared with various idealised theoretical models. This approach has several advantages. First, it is entirely analogous to the multiple exponential expansion used to extract longitudinal and transverse relaxation times. This suggests that, provided sufficient data points is acquired in the $\Delta$ dimension, a continuous distribution of $\mathrm{D}(\mathrm{q})$ could be measured with exponential deconvolution packages such as CONTIN or WINDXP. A second advantage is that the coefficients $D_{n}(q)$ are useful in establishing empirical correlations with non-NMR properties such as rheological or sensory parameters. This is analogous to relaxation -timesensory or rheological correlations. The third advantage is the ease of comparison with analytical models. For example, comparison of the $\mathrm{p}_{\mathrm{n}}$ and $\mathrm{D}_{\mathrm{n}}(\mathrm{q})$ coefficients shows that the data for flocculated and unflocculated, non-creaming emulsions [1] is best described by a standard two-compartment exchange model [2] where water exchanges between the inside and outside of the flocs. In the limit of low floc water content and low diffusivity inside the flocs we find that $\mathrm{p}_{0}(\mathrm{q})=1$ and $\mathrm{D}_{0}(\mathrm{q})=\mathrm{D}_{\mathrm{b}} \mathrm{q}^{2} /\left[1+\mathrm{Cq}^{2}\right]$ where $\mathrm{D}_{\mathrm{b}}$ is the effective diffusion coefficient of water inside the flocs and $\mathrm{C}$ is related to the exchange rate as $D_{b} /\left(k_{b}-k_{a}\right)$. The exchange rate is observed to decrease with increasing emulsion concentration presumably because of the larger number and size of flocs. Large differences are also found for flocculated and unflocculated emulsions.

In contrast, the data for concentrated creams [1] are best described by an anomalous diffusion model, such that,

$$
\mathrm{S}(\mathrm{q}, \Delta) / \mathrm{S}(0, \Delta)=\mathrm{p}_{0}(\mathrm{q}) \exp \left[-\mathrm{D}_{0}(\mathrm{q}) \Delta^{\mathrm{d}}\right]
$$

where $\mathrm{d}$ is a dimensionality parameter. Both the dimensionality, $\mathrm{d}$, and the diffusivity parameter, $\mathrm{D}_{0}(\mathrm{q})$, are found to decrease with increasing oil volume fraction, though the reasons for these trends remain unclear.

\section{NMR correlations with rheology}

The NMR-rheology connection has been explored [1] by fixing the wavevector, $q$, at the value, $\mathrm{q}_{0}$, for which the dimensionality exponent for the hexadecane creams, $d\left(q_{0}\right)$, is unity. The echo attenuation data then conforms to the single exponential expression, $\exp \left[-\mathrm{D}_{0}\left(\mathrm{q}_{0}\right) \Delta\right]$ and it is possible to compare the diffusion rate, $\mathrm{D}_{0}\left(\mathrm{q}_{0}\right)$, with the bulk rheological parameters. The data for all the emulsions then conforms to the relationships,

$$
\mathrm{D}_{0}\left(\mathrm{q}_{0}\right) / \mathrm{D}_{0}\left(\mathrm{q}_{0}\right)_{\text {water }}=-\mathrm{aLn}\left[\eta / \eta_{\text {water }}\right]+\mathrm{b}
$$

and

$$
\mathrm{D}_{0}\left(\mathrm{q}_{0}\right) / \mathrm{D}_{0}\left(\mathrm{q}_{0}\right)_{\text {water }}=\mathrm{cG}^{\prime-\mathrm{e}} \text { or } \mathrm{fG}^{\prime \prime-h}
$$

where $G$ is either $G^{\prime}$ or $G^{\prime \prime}$ and a,b,c,e,f and $h$ are constants The fundamental reasons for these empiric relationships also remain to be explored. 
Future work will focus on the kinetics of the flocculation and creaming processes by using q-space weighted imaging [2]. In this way it should be possible to monitor the space-time dependence of the continuous phase diffusion propagator in creaming and freezing emulsions.

\section{References}

[1] Hills B, Manoj P, Destruel C. NMR q-space microscopy of concentrated oil-in-water emulsions. Magnetic Resonance Imaging, 2000; 18:319-333.

[2] Hills BP. Magnetic Resonance Imaging in Food Science. John Wiley, and Sons, 1998. 